\title{
Photobiomodulation Therapy (PBMT) in Peripheral Nerve Regeneration: A Systematic Review
}

\author{
Marcelie Priscila de Oliveira Rosso ${ }^{1}$, Daniela Vieira Buchaim ${ }^{2,3}$, Natália Kawano ${ }^{2}$, \\ Gabriela Furlanette ${ }^{2}$, Karina Torres Pomini ${ }^{1}$ and Rogério Leone Buchaim ${ }^{1,2, *}$ \\ 1 Department of Biological Sciences (Anatomy), Bauru School of Dentistry, University of São Paulo (USP), \\ Alameda Dr. Octávio Pinheiro Brisola 9-75, Vila Nova Cidade Universitária, Bauru, São Paulo CEP \\ 17012-901, Brazil; marcelierosso@usp.br (M.P.d.O.R.); karinatorrespomini@gmail.com (K.T.P.) \\ 2 Medical School, Discipline of Human Morphophysiology, University of Marilia (UNIMAR), \\ Av. Higino Muzi Filho, 1001 Campus Universitário, Jardim Araxa, Marília, São Paulo CEP 17525-902, Brazil; \\ danibuchaim@usp.br (D.V.B.); natalia.kawano@hotmail.com (N.K.); gafurla@hotmail.com (G.F.) \\ 3 Medical School, Discipline of Neuroanatomy, University Center of Adamantina (UNIFAI), \\ Rua Nove de Julho, 730, Centro, Adamantina, São Paulo CEP 17800-000, Brazil \\ * Correspondence: rogerio@fob.usp.br; Tel.: +55-14-3235-8226
}

Received: 17 May 2018; Accepted: 7 June 2018; Published: 9 June 2018

\begin{abstract}
Photobiomodulation therapy (PBMT) has been investigated because of its intimate relationship with tissue recovery processes, such as on peripheral nerve damage. Based on the wide range of benefits that the PBMT has shown and its clinical relevance, the aim of this research was to carry out a systematic review of the last 10 years, ascertaining the influence of the PBMT in the regeneration of injured peripheral nerves. The search was performed in the PubMed/MEDLINE database with the combination of the keywords: low-level laser therapy AND nerve regeneration. Initially, 54 articles were obtained, 26 articles of which were chosen for the study according to the inclusion criteria. In the qualitative aspect, it was observed that PBMT was able to accelerate the process of nerve regeneration, presenting an increase in the number of myelinated fibers and a better lamellar organization of myelin sheath, besides improvement of electrophysiological function, immunoreactivity, high functionality rate, decrease of inflammation, pain, and the facilitation of neural regeneration, release of growth factors, increase of vascular network and collagen. It was concluded that PBMT has beneficial effects on the recovery of nerve lesions, especially when related to a faster regeneration and functional improvement, despite the variety of parameters.
\end{abstract}

Keywords: low-level laser therapy; nerve regeneration; peripheral nerve repair; photobiomodulation therapy; tissue regeneration

\section{Introduction}

Low-level laser therapy (LLLT), now commonly referred to as photobiomodulation therapy (PBMT), using low-level infrared light spectrum lasers is considered a therapeutic advance. Its effects are related to tissue biostimulation, presenting therapeutic responses from photoelectric, photoenergetic, and photochemical reactions [1]. Scientific research has shown the application of PBMT in bone tissue and peripheral nerves with good results whether or not it is associated with other supporting methods in tissue repair [2-7].

Laser photobiomodulation presents itself as an electromagnetic technology that is being inserted into clinical practice due to its characteristics that differ from other conventional thermal sources $[8,9]$. It was observed that there are several features of PBMT that are related to the reduction of tissue repair time and its capacity to increase cell proliferation [10]. 
In rehabilitative health, PBMT was inserted to promote the repair and recovery of tissues. For example, in physical therapy, the use of PBMT is applied in postoperative phases as an aid in the muscular, nervous, joint, and other functional recovery processes, and in dentistry it is applied in the processes of dental extraction, grafting, osteonecrosis, and periodontal lesions [11-13].

The wavelength of infrared irradiation is easily absorbed by tissues and the loss of intensity is minimal, affecting metabolic modifications, DNA activity, adenosine triphosphate (ATP) formation, and the mitochondrial chain. The effect of photobiomodulation is due to the absorption of the photons by cytochrome $\mathrm{C}$ oxidase in the mitochondrial respiratory chain, consequently increasing the cytochrome $\mathrm{C}$ oxidase activity and therefore ATP formation. ATP from injured or regions of impaired blood perfusion can reactivate injured cells and metabolic disorders [10]. PBMT is also related to pain and inflammation relief and prevention of tissue death to avoid neurological degeneration [14,15].

The wavelength is the key point that regulates the depth and penetration of the laser irradiance in the tissue, noting that the absorption and dispersion coefficients are larger at the lower wavelengths. Regarding the type of wave, whether continuous or pulsed, there are still divergences in which is the best and for which factors are the pulse parameters to be chosen [16]. PBMT presents difficulties in selecting the most suitable parameters for its application due to the lack of standardization, since wavelength, power density, irradiation time, and light polarization have repercussions on the biological effects [9].

Due to the photochemical and photobiological effects of PBMT at the cellular level, there is a relationship between the improvement of trophic conditions and the reduction of inflammatory processes, closely related to a more efficient nervous regeneration and, also, promoting the secretion of neural factors $[16,17]$. Thus, photobiomodulation therapy in the neurological area acts as an adjuvant in the treatment of traumatic brain degeneration/injury, spinal cord trauma, and in the process of peripheral nerve regeneration.

Peripheral nerve lesions are a reality today, but there is a deficit in relating effective treatments for recovery of the nerves, resulting in considerable functional changes in the daily life of the individual. When injured, the nerve can lose its function, causing motor or sensitive deficits. There is retrograde axonal degeneration to the area of the lesion, so regeneration occurs slowly and sometimes incompletely $[18,19]$.

At the end of the $80^{\prime}$ s, the scientific interest in the therapeutic approach of rehabilitation for neural lesions was initiated [20], due to the good results with the use of PBMT in the recovery of injured peripheral nerves but, until the present day, there are still difficulties related to the application parameters $[19,20]$. Its beneficial effects are independent of the repair technique, neurorrhaphy techniques, and the use of fibrin sealants $[3,6,7,21]$.

PBMT leads to changes in important vascular levels such as elevation of the secretion of antiapoptotic factors in ischemic organs, providing a better wound healing [22,23]; the presence of angiogenesis when ischemic organs were injured [24,25]; a decrease in the site of infarction in rats; as well as elevation in neurological scores following embolic stroke in rats [26,27].

Due to the high range of benefits that PBMT has shown and its clinical relevance of application, the aim of this research was to carry out a systematic review of the scientific papers published in the last 10 years verifying the relation of PBMT with the regeneration of injured peripheral nerves.

\section{Materials and Methods}

A search was performed in the PubMed/MEDLINE database, combining low-level laser therapy AND nerve regeneration keywords, over the last 10 years and restricted to the English language. The next step was to restrict the verification and consultation of articles that used animals as a study object (non-human species).

We verified those articles that presented titles and summaries that approached the subject of this research, as well as methodology, results, and relevance for its practical application. 
The articles included should necessarily be presented with full access to the text. The acquired texts were analyzed and synthesized in a reflexive way in order to obtain consistent information on the subject.

\section{Results}

Initially, 54 articles were obtained from the PubMed/MEDLINE database, of which 28 were excluded because they were not included in the search criteria (in English, study in animals, and full access to content). At the end, 26 articles related to the subject were included. Figure 1 schematizes the search system, according to PRISMA Flow Diagram [28].
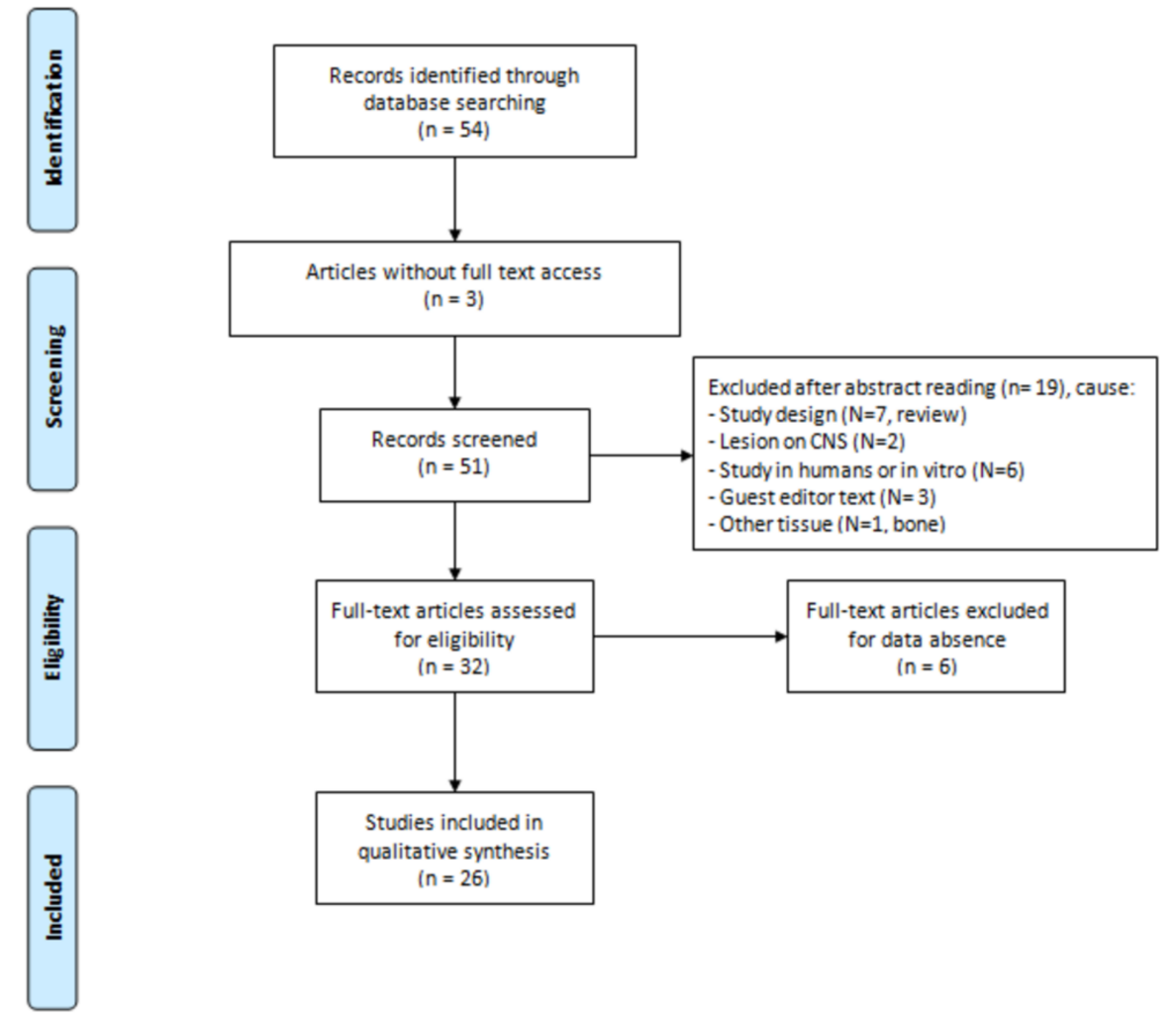

Figure 1. Design used to select the articles.

Table 1 summarizes the data presented in the 26 articles selected for this research. 
Table 1. Data of selected articles.

\begin{tabular}{|c|c|c|c|c|c|c|c|c|c|}
\hline Authors & $\begin{array}{l}\text { Type of Laser } \\
\text { (Manufacturer) }\end{array}$ & $\begin{array}{l}\text { Wavelength } \\
(\mathrm{nm}) / \mathrm{Spot} \\
\text { Beam }\end{array}$ & Energy $(\mathrm{mW})$ & $\begin{array}{l}\text { Energy } \\
\text { Density } \\
\left(\mathrm{J} / \mathrm{cm}^{2}\right)\end{array}$ & $\begin{array}{c}\text { Radiation } \\
\text { Amount }\end{array}$ & Variables & Irradiation Site & Evaluation Time & Main Results \\
\hline $\begin{array}{l}\text { Buchaim et al. } \\
{[2]}\end{array}$ & $\begin{array}{l}\text { GaAlAs (Laserpulse } \\
\text { IBRAMED, Brazil) }\end{array}$ & $660 / 0.116$ & 30 & 4 & $\begin{array}{l}16 \text { s per point; } \\
3 \text { points }\end{array}$ & $\begin{array}{l}\text { Sural nerve graft was coapted to the } \\
\text { vagus nerve using the fibrin glue. }\end{array}$ & Right side of the neck. & $\begin{array}{l}\text { Application on the 1st } \\
\text { day post-operatory, } 5 \\
\text { weeks, } 3 \text { times a week. } \\
\text { Evaluation } 30 \text { days } \\
\text { after irradiation. }\end{array}$ & $\begin{array}{l}\text { LLLT improved the nerve } \\
\text { regeneration. }\end{array}$ \\
\hline $\begin{array}{l}\text { Buchaim et al. } \\
{[3]}\end{array}$ & $\begin{array}{l}\text { GaAlAs (Laserpulse } \\
\text { IBRAMED, Brazil) }\end{array}$ & $830 / 0.116$ & 30 & 6 & $\begin{array}{l}24 \text { s per point; } \\
3 \text { points }\end{array}$ & $\begin{array}{l}\text { Neurotmeses of buccal branch of } \\
\text { facial nerve, followed by end-to-end } \\
\text { suture or coaptation with } \\
\text { heterologous fibrin sealant derived } \\
\text { from snake venom }\end{array}$ & $\begin{array}{l}\text { On the surgical site, on } \\
\text { both sides of the face }\end{array}$ & $\begin{array}{l}\text { Application 1st day } \\
\text { post-operatory, } 3 \\
\text { times /week for } 5 \\
\text { weeks. Evaluation } 5 \\
\text { and } 10 \text { weeks after } \\
\text { the surgery. }\end{array}$ & $\begin{array}{l}\text { LLLT showed satisfactory results } \\
\text { on facial nerve regeneration. }\end{array}$ \\
\hline $\begin{array}{l}\text { Buchaim et al. } \\
{[6]}\end{array}$ & $\begin{array}{l}\text { GaAlAs (Laserpulse } \\
\text { IBRAMED }^{\circledR} \text {, Brazil) }\end{array}$ & $830 / 0.116$ & 30 & 6.2 & $\begin{array}{l}24 \text { s per point; } \\
3 \text { points }\end{array}$ & $\begin{array}{l}\text { Neurotmeses of buccal branch of } \\
\text { facial nerve, end-to-end anastomosis. } \\
\text { Use of epineural suture or } \\
\text { coaptation with heterologous fibrin } \\
\text { sealant derived from snake venom. }\end{array}$ & $\begin{array}{l}\text { On the surgical site, on } \\
\text { both sides of the face }\end{array}$ & $\begin{array}{l}\text { Application on the 1st } \\
\text { day post-operatory, } 3 \\
\text { times a week, for } \\
5 \text { weeks. }\end{array}$ & $\begin{array}{l}\text { Laser stimulated axonal } \\
\text { regeneration accelerated the } \\
\text { process of functional recovery of } \\
\text { whisker, and the two techniques } \\
\text { used allowed the growth of } \\
\text { axons. }\end{array}$ \\
\hline Rosso et al. [7] & $\begin{array}{l}\text { GaAlAs (Laserpulse } \\
\text { IBRAMED }^{\circledR}, \text { Brazil) }\end{array}$ & $830 / 0.116$ & 30 & 6.2 & $\begin{array}{l}24 \text { s per point; } \\
3 \text { points }\end{array}$ & $\begin{array}{l}\text { Neurotmeses in buccal branch of } \\
\text { facial nerve, end-lateral anastomosis } \\
\text { in the zygomatic branch of the facial } \\
\text { nerve with epineural suture or } \\
\text { heterologous sealant of fibrin } \\
\text { derived from snake venom. }\end{array}$ & $\begin{array}{l}\text { On the surgical site, on } \\
\text { both sides of the face }\end{array}$ & $\begin{array}{l}\text { Application on the 1st } \\
\text { day post- operatory, } 3 \\
\text { times a week, for } \\
10 \text { weeks. }\end{array}$ & $\begin{array}{l}\text { Laser groups presented faster } \\
\text { functional recovery, similar } \\
\text { results to the control group. It } \\
\text { was observed that PBMT } \\
\text { provided accelerated } \\
\text { morphological and functional } \\
\text { repair in the two techniques } \\
\text { used. }\end{array}$ \\
\hline $\begin{array}{l}\text { Ziago et al. } \\
\quad[19]\end{array}$ & $\begin{array}{c}\text { GaAlAs (Twin } \\
\text { Laser, MMO, São } \\
\text { Carlos, SP, Brazil) }\end{array}$ & $780 / 0.04$ & 40 & $\begin{array}{c}4 \\
10 \\
50\end{array}$ & $\begin{array}{l}4,10 \text { e } 50 \text { s per } \\
\text { point; } 3 \text { points }\end{array}$ & Crushing of the left sciatic nerve. & On the surgical site & $\begin{array}{l}\text { Application during } 6 \\
\text { sessions on } \\
\text { alternate days. }\end{array}$ & $\begin{array}{l}\text { Best morphological quantitative } \\
\text { and morphometric results on } \\
\text { L10 group after } 15 \text { days of nerve } \\
\text { lesion. }\end{array}$ \\
\hline $\begin{array}{l}\text { Alessi Pissulin } \\
\text { et al. [29] }\end{array}$ & $\begin{array}{l}\text { GaAs (Endophoton, } \\
\text { KLD Biosystems, } \\
\text { Amparo, Brazil) }\end{array}$ & $904 / 0.035$ & 50 & 69 & 48 s per point & $\begin{array}{l}0.5 \% \text { bupivacaine injection to the } \\
\text { right and } 0.9 \% \text { sodium chloride } \\
\text { injection to the left on } \\
\text { sternocleidomastoid muscle and } \\
\text { accessory nerve exposed in surgery. }\end{array}$ & Ventral side of the neck & $\begin{array}{l}\text { Application 1st day } \\
\text { post-operatory, during } \\
5 \text { successive days. }\end{array}$ & $\begin{array}{l}\text { LLLT reduced the aggressive } \\
\text { effects of bupivacaine on the } \\
\text { nerve and the muscle, of } \\
\text { muscular degeneration, of } \\
\text { myonecrosis and fibrosis, kept } \\
\text { the morphology of the axon and } \\
\text { the myelin sheath. }\end{array}$ \\
\hline $\begin{array}{l}\text { Takhtfooladi; } \\
\text { Sharifi [30] }\end{array}$ & $\begin{array}{l}\text { GaAlAs (pulsed) } \\
\text { LED (red and blue) } \\
\quad(-)\end{array}$ & $\begin{array}{c}680 / 0.04 \\
650 / 1.5 \text { red } \\
450 / 1.5 \text { blue }\end{array}$ & 10 & 10 & $\begin{array}{c}200 \text { s per } \\
\text { point; } 3 \text { points }\end{array}$ & $\begin{array}{l}\text { Neurotmeses of right sciatic nerve } \\
\text { followed by epineural neurorrhaphy. }\end{array}$ & $\begin{array}{l}\text { On the surgical site, } \\
\text { sciatic nerve }\end{array}$ & $\begin{array}{l}\text { Application 1st day } \\
\text { post-operatory, during } \\
14 \text { successive days }\end{array}$ & $\begin{array}{l}\text { LLLT increased Schwann cells on } \\
\text { the great myelinic axons and on } \\
\text { neurons, sped up and } \\
\text { potentialized nerve regeneration. }\end{array}$ \\
\hline
\end{tabular}


Table 1. Cont.

\begin{tabular}{|c|c|c|c|c|c|c|c|c|c|}
\hline Authors & $\begin{array}{c}\text { Type of Laser } \\
\text { (Manufacturer) }\end{array}$ & $\begin{array}{c}\text { Wavelength } \\
(\mathrm{nm}) / \text { Spot } \\
\text { Beam }\end{array}$ & Energy $(\mathrm{mW})$ & $\begin{array}{l}\text { Energy } \\
\text { Density } \\
\left(\mathrm{J} / \mathrm{cm}^{2}\right)\end{array}$ & $\begin{array}{c}\text { Radiation } \\
\text { Amount }\end{array}$ & Variables & Irradiation Site & Evaluation Time & Main Results \\
\hline $\begin{array}{l}\text { Takhtfooladi } \\
\text { et al. [31] }\end{array}$ & $\begin{array}{l}\text { InGaAlP (Teralaser; } \\
\text { DMC®São Carlos, } \\
\text { SP, Brazil) }\end{array}$ & $685 / 0.028$ & 15 & 3 & 10 s per point & Crushing of the left sciatic nerve. & $\begin{array}{l}\text { On the surgery site on } \\
\text { sciatic nerve. }\end{array}$ & $\begin{array}{l}\text { Application on the 1st } \\
\text { day post-operatory, } \\
\text { during } 21 \\
\text { successive days. }\end{array}$ & $\begin{array}{l}\text { LLLT accelerated and improved } \\
\text { the nerve function after } \\
\text { crushing lesion. }\end{array}$ \\
\hline $\begin{array}{l}\text { Wang et al. } \\
\text { [32] }\end{array}$ & $\begin{array}{l}\text { GaAlAs (Transverse } \\
\text { IND. CO., LTD., } \\
\text { Taipei, Taiwan) }\end{array}$ & $808 / 3.8$ & 170 & $\begin{array}{l}3 \\
8 \\
15\end{array}$ & $\begin{array}{c}67.2 \mathrm{~s} \\
179 \mathrm{~s} \\
335.6 \mathrm{~s}\end{array}$ & Crushing of the right sciatic nerve. & On lesion on sciatic nerve. & $\begin{array}{l}\text { Application during } 20 \\
\text { successive days. }\end{array}$ & $\begin{array}{l}\text { LLLT }\left(3 \text { and } 8 \mathrm{~J} / \mathrm{cm}^{2}\right) \text { accelerated } \\
\text { functional and morphologic } \\
\text { recovery of the nerve, increased } \\
\text { the expression of the } \\
\text { marker GAP43. }\end{array}$ \\
\hline $\begin{array}{l}\text { Shen; Yang; } \\
\text { Liu [33] }\end{array}$ & $\begin{array}{c}\text { AlGaInP } \\
\text { (Megalas1-AM-800, } \\
\text { Konftec Co., Taipei, } \\
\text { Taiwan, ROC) }\end{array}$ & $660 /-$ & 0.0032 & 3.84 & 5 min per day & $\begin{array}{l}\text { Neurotmeses of the left sciatic nerve, } \\
10 \text { mm gap and use of biodegradable } \\
\text { tube containing genipin-cross-linked } \\
\text { gelatin annexed with } \beta \text {-tricalcium } \\
\text { phosphate ceramic particles } \\
\text { (genipin-gelatin-tricalcium } \\
\text { phosphate, GGT) }\end{array}$ & Applied to the surgical site. & $\begin{array}{l}\text { Application 1st day } \\
\text { post-operatory, during } \\
20 \text { successive days. } \\
\text { Euthanasia after } \\
8 \text { weeks. }\end{array}$ & $\begin{array}{l}\text { LLLT obtained better functional, } \\
\text { electrophysiological and } \\
\text { histomorphometric results and } \\
\text { assisted on neural repair. }\end{array}$ \\
\hline $\begin{array}{l}\text { Shen; Yang; } \\
\text { Liu [34] }\end{array}$ & $\begin{array}{c}\text { AlGaInP } \\
\text { (MegalasVR } \\
\text {-AM-800; Konftec, } \\
\text { Taipei, Taiwan) }\end{array}$ & $660 /-$ & 50 & $\begin{array}{c}\text { Immediate } \\
\text { post-surgery } \\
(5.76) \\
9 \text { following } \\
\text { days }(0.96)\end{array}$ & $\begin{array}{l}\text { Immediate } \\
\text { post-surgery } \\
(30 \text { min) } \\
9 \text { successive } \\
\text { consecutive (5 } \\
\text { min) }\end{array}$ & $\begin{array}{l}\text { Neurotmeses of the left sciatic nerve, } \\
15 \text { mm gap and the use of } \\
\text { 1-ethyl-3-(3-dimethylaminopro-pyl) } \\
\text { carbodiimide (EDC) cross-linked } \\
\text { gelatin, annexed with } \beta \text {-tricalcium } \\
\text { phosphate (TCP) ceramic particles } \\
\text { (EDC-Gelatin-TCP, EGT). }\end{array}$ & On the surgery site. & $\begin{array}{l}\text { Application } \\
\text { immediately after the } \\
\text { lesion, during } 9 \\
\text { successive days. } \\
\text { Euthanasia after } \\
12 \text { weeks. }\end{array}$ & $\begin{array}{l}\text { LLLT showed better results on } \\
\text { the functional index, on } \\
\text { development, on } \\
\text { electrophysiology, on nerve } \\
\text { regeneration, larger neural tissue } \\
\text { area, larger axon, and myelin } \\
\text { sheath diameter. }\end{array}$ \\
\hline $\begin{array}{l}\text { Medalha et al. } \\
{[35]}\end{array}$ & $\begin{array}{l}\text { GaAlAs (Teralaser, } \\
\text { DMC São Carlos, } \\
\text { São Paulo, Brazil) }\end{array}$ & $\begin{array}{l}660 / 0.028 \\
808 / 0.028\end{array}$ & $\begin{array}{l}30 \\
30\end{array}$ & $\begin{array}{l}10 \text { e } 50 \\
10 \text { e } 50\end{array}$ & $\begin{array}{l}9 \mathrm{~s} \text { and } 47 \mathrm{~s} ; \\
3 \text { points } \\
9 \mathrm{~s} \text { and } 47 \mathrm{~s} \\
3 \text { points }\end{array}$ & $\begin{array}{l}\text { Neurotmeses of the sciatic nerve, } \\
\text { approximately } 3 \mathrm{~mm} \text { distal to the } \\
\text { tendon of the internal obturator. } \\
\text { Anastomosis with } 3 \text { sutures using } \\
\text { nylon monofilament } 10-0 .\end{array}$ & Applied to the surgical site. & $\begin{array}{l}\text { Application 1st day } \\
\text { post-operatory during } 5 \\
\text { successive days and } 2 \\
\text { days interval until } \\
\text { completing } 15 \text { days. }\end{array}$ & $\begin{array}{l}\text { LLLT } 808 \mathrm{~nm} \text { on } 50 \mathrm{~J} / \mathrm{cm}^{2} \\
\text { obtained higher fiber density. } \\
\text { LLLT } 660 \mathrm{~nm} \text { on } 50 \mathrm{~J} / \mathrm{cm}^{2} \\
\text { presented larger diameters of } \\
\text { axons and of fibers of gait } \\
\text { functional recovery. }\end{array}$ \\
\hline Shen et al. [36] & $\begin{array}{c}\text { GaAlAsP } \\
\text { (Aculas-AM-100A, } \\
\text { Konftec Co., Taipei, } \\
\text { Taiwan) }\end{array}$ & $660 / 0.1$ & 50 & 2 & $\begin{array}{l}2 \text { min per day; } \\
2 \text { points at the } \\
\text { same time }\end{array}$ & $\begin{array}{l}\text { A biodegradable nerve conduit } \\
\text { containing genipin-cross-linked } \\
\text { gelatin was annexed using } \\
\text { beta-tricalcium phosphate (TCP) } \\
\text { ceramic particles } \\
\text { (genipin-gelatin-TCP, GGT) with a } \\
15 \mathrm{~mm} \text { sciatic nerve transection gap. }\end{array}$ & On the sciatic nerve. & $\begin{array}{l}\text { Application 1st day } \\
\text { post-operatory during } \\
10 \text { successive days. }\end{array}$ & $\begin{array}{l}\text { LLLT accelerated the nerve } \\
\text { regeneration due to the larger } \\
\text { neural tissue, larger diameter } \\
\text { and thicker melin sheath, motor } \\
\text { function, electrophysiology and } \\
\text { muscular innervation. }\end{array}$ \\
\hline
\end{tabular}


Table 1. Cont.

\begin{tabular}{|c|c|c|c|c|c|c|c|c|c|}
\hline Authors & $\begin{array}{l}\text { Type of Laser } \\
\text { (Manufacturer) }\end{array}$ & $\begin{array}{c}\text { Wavelength } \\
(\mathrm{nm}) / \mathrm{Spot} \\
\text { Beam }\end{array}$ & Energy $(\mathrm{mW})$ & $\begin{array}{l}\text { Energy } \\
\text { Density } \\
\left(\mathrm{J} / \mathrm{cm}^{2}\right)\end{array}$ & $\begin{array}{c}\text { Radiation } \\
\text { Amount }\end{array}$ & Variables & Irradiation Site & Evaluation Time & Main Results \\
\hline $\begin{array}{l}\text { Chen et al. } \\
\text { [37] }\end{array}$ & $\begin{array}{l}\text { GaAlAs (Transverse } \\
\text { IND. CO., LTD., } \\
\text { Taipei, Taiwan) }\end{array}$ & $808 \pm 5 / \leq 0.5$ & 190 & 8 & $207 \mathrm{~s}$ & $\begin{array}{l}\text { Chronic compression on dorsal root } \\
\text { ganglion. A thin L shaped needle } \\
(0,6 \mathrm{~mm} \text { of diameter) was inserted } 4 \\
\mathrm{mm} \text { in the L4 and L5 intervertebral } \\
\text { foramen. }\end{array}$ & $\begin{array}{l}\text { On the dorsal root of L4 } \\
\text { and L5. }\end{array}$ & $\begin{array}{l}\text { Application } 1 \text { st day } \\
\text { post-operatory, per } 8 \\
\text { successive days. } \\
\text { Euthanasia } 4 \text { e } 8 \text { days. }\end{array}$ & $\begin{array}{l}\text { LLLT decreased the levels of } \\
\text { inflammatory cytokines and of } \\
\text { pain, facilitating the nerve } \\
\text { regeneration, demonstrated by } \\
\text { levels of TNF-a, IL-1b e GAP-43. }\end{array}$ \\
\hline $\begin{array}{l}\text { Belchior et al. } \\
\quad[38]\end{array}$ & $\begin{array}{l}\text { GaAlAs (KLD } \\
\text { Endophoton model) }\end{array}$ & $660 / 0.63$ & 26.3 & 4 & $96.7 \mathrm{~s} ; 3$ points & Crushing of the right sciatic nerve. & On the surgical site. & $\begin{array}{l}\text { Application 1st day } \\
\text { post-operatory, during } \\
20 \text { successive days. }\end{array}$ & $\begin{array}{l}\text { LLLT was positive on the } \\
\text { functional index after the } \\
\text { 21st day. }\end{array}$ \\
\hline $\begin{array}{c}\text { Barbosa et al. } \\
\text { [39] }\end{array}$ & $\begin{array}{l}\text { GaAlAs (Ibramed } \\
\text { Equipamentos } \\
\text { Médicos) }\end{array}$ & $\begin{array}{l}660 / 0.06 \\
830 / 0.116\end{array}$ & 30 & $\begin{array}{l}10 \\
10\end{array}$ & $\begin{array}{c}20 \mathrm{~s} \\
38.66 \mathrm{~s}\end{array}$ & Crushing of the right sciatic nerve. & On the surgical site. & $\begin{array}{l}\text { Application 1st day } \\
\text { post-operatory, during } \\
20 \text { successive days. }\end{array}$ & $\begin{array}{l}\text { LLLT } 660 \mathrm{~nm} \text { promoted } \\
\text { functional recovery in a faster } \\
\text { manner. }\end{array}$ \\
\hline $\begin{array}{l}\text { Marcolino et } \\
\text { al. [40] }\end{array}$ & $\begin{array}{l}\text { AlGaAs (Laser } \\
\text { Diode, Ibramed) }\end{array}$ & $830 / 0.116$ & 30 & $\begin{array}{l}10 \\
40 \\
80\end{array}$ & $\begin{array}{l}38.66 \mathrm{~s} \\
154.66 \mathrm{~s} \\
309.33 \mathrm{~s}\end{array}$ & Crushing of the right fibular nerve. & On the right sciatic nerve. & $\begin{array}{l}\text { Application } \\
\text { immediately after } \\
\text { surgery and during the } \\
21 \text { successive days. }\end{array}$ & $\begin{array}{l}40 \mathrm{~J} / \mathrm{cm}^{2} \text { and } 80 \mathrm{~J} / \mathrm{cm}^{2} \mathrm{LLLT} \\
\text { influenced the functional } \\
\text { recovery of the nerve. }\end{array}$ \\
\hline $\begin{array}{l}\text { Akgul; } \\
\text { Gulsoy; } \\
\text { Gulcur [41] }\end{array}$ & $\begin{array}{l}\text { Laser diode (model: } \\
\text { DH650-24-3(5), } \\
\text { Huanic, China) }\end{array}$ & $650 / \approx 0.14$ & 25 & 10 & $\begin{array}{l}57 \mathrm{~s} \text { on } \\
3 \text { points }\end{array}$ & Crushing of the sciatic nerve. & On the sciatic nerve. & $\begin{array}{l}\text { Early group: } \\
\text { Application after } \\
\text { surgery, up to the 14th } \\
\text { day. Delayed group: } \\
\text { Application on the } 7 \text { th } \\
\text { day post-operatory and } \\
\text { up to the 21st day. }\end{array}$ & $\begin{array}{l}\text { LLLT accelerated nervous } \\
\text { recovery. The group with } \\
\text { delayed application showed } \\
\text { better functional results. }\end{array}$ \\
\hline $\begin{array}{l}\text { Gigo-Benato } \\
\text { et al. [42] }\end{array}$ & $\begin{array}{l}\text { GaAlAs (TWIN } \\
\text { LASER; MM Optics, } \\
\text { São Carlos, SP, } \\
\text { Brazil) }\end{array}$ & $\begin{array}{l}660 / 0.04 \\
780 / 0.04\end{array}$ & $\begin{array}{l}40 \\
40\end{array}$ & $\begin{array}{l}10,60 \text { and } 120 \\
10,60 \text { and } 120\end{array}$ & $\begin{array}{l}0.3 \mathrm{~s}, 1 \mathrm{~min} \\
\text { and } 2 \mathrm{~min} \\
0.3 \mathrm{~s}, 1 \mathrm{~min} \\
\text { and } 2 \mathrm{~min} ; 2 \\
\text { points }\end{array}$ & Crushing of the left sciatic nerve. & Applied to the surgical site. & $\begin{array}{l}\text { Application 1st day } \\
\text { post-operatory, during } \\
10 \text { successive days. }\end{array}$ & $\begin{array}{l}\mathrm{LLLT}\left(660 \mathrm{~nm}, 10 \mathrm{~J} / \mathrm{cm}^{2} \text { or } 60\right. \\
\left.\mathrm{J} / \mathrm{cm}^{2}\right) \text { accelerated the } \\
\text { neuromuscular recuperation. }\end{array}$ \\
\hline $\begin{array}{c}\text { dos Reis et al. } \\
{[43]}\end{array}$ & $\begin{array}{c}\text { AlGaAs (KLD } \\
\text { Endophoton model) }\end{array}$ & $660 / 0.63$ & 26.3 & 4 & $\begin{array}{c}96.7 \mathrm{~s} \mathrm{per} \\
\text { point; } 3 \text { points }\end{array}$ & $\begin{array}{l}\text { Neurotmeses and epineural } \\
\text { anastomosis on the right sciatic } \\
\text { nerve. }\end{array}$ & On the surgical site. & $\begin{array}{l}\text { Application 1st day } \\
\text { post-operatory, } 20 \\
\text { successive days. }\end{array}$ & $\begin{array}{l}\text { LLLT significantly changed the } \\
\text { morphometry (myelin sheath), } \\
\text { but did not interfere on } \\
\text { functionality. }\end{array}$ \\
\hline $\begin{array}{l}\text { Yang et al. } \\
\text { [44] }\end{array}$ & $\begin{array}{c}\text { GaAlAs } \\
\text { (Aculas-Am series, } \\
\text { Multi-channel LLLT } \\
\text { System, Konftec } \\
\text { Corp., Taipei, } \\
\text { Taiwan) }\end{array}$ & $660 / \approx 0.2$ & 30 & 9 & $\begin{array}{l}60 \text { s per point; } \\
4 \text { points }\end{array}$ & $\begin{array}{l}\text { Use of Mesenchymal stem cells } \\
\text { (MSC) on the lesion by crushing of } \\
\text { sciatic nerve. }\end{array}$ & On the sciatic nerve & 7 successive days. & $\begin{array}{l}\text { LLLT+MSC improved the } \\
\text { electrophysiologic function, S100 } \\
\text { immunoreactivity, less } \\
\text { inflammatory cells and less } \\
\text { vacuole formation. }\end{array}$ \\
\hline $\begin{array}{c}\text { de Oliveira } \\
\text { Martins et al. } \\
\quad[45]\end{array}$ & $\begin{array}{l}\text { GaAs } \\
\text { (Laserpulse-Laser, } \\
\text { Ibramed Brazil) } \\
\text { pulsado }\end{array}$ & $904 / 0.1$ & $70 \mathrm{Wpk}$ & 6 & $\begin{array}{l}18 \text { s on } \\
5 \text { points }\end{array}$ & $\begin{array}{l}\text { Pulsed LLLT. } \\
\text { Lesion on alveolar nerve, by a } \\
\text { hemostatic Crile clamp. }\end{array}$ & On the sciatic nerve. & $\begin{array}{l}10 \text { sessions every } \\
10 \text { days. }\end{array}$ & $\begin{array}{l}\text { LLLT obtained better } \\
\text { nociception, higher expression of } \\
\text { neural growth factor (NGF) } 53 \% \\
\text { and of expression of } \\
\text { neurotrophic factor (BDNF) } 40 \% \text {. }\end{array}$ \\
\hline
\end{tabular}


Table 1. Cont.

\begin{tabular}{|c|c|c|c|c|c|c|c|c|c|}
\hline Authors & $\begin{array}{l}\text { Type of Laser } \\
\text { (Manufacturer) }\end{array}$ & $\begin{array}{l}\text { Wavelength } \\
(\text { nm)/Spot } \\
\text { Beam }\end{array}$ & Energy $(\mathrm{mW})$ & $\begin{array}{l}\text { Energy } \\
\text { Density } \\
\left(\mathrm{J} / \mathrm{cm}^{2}\right)\end{array}$ & $\begin{array}{c}\text { Radiation } \\
\text { Amount }\end{array}$ & Variables & Irradiation Site & Evaluation Time & Main Results \\
\hline $\begin{array}{l}\text { Gomes; } \\
\text { Dalmarco; } \\
\text { André [46] }\end{array}$ & $\mathrm{HeNe}(-)$ & $632.8 / 0.1$ & 5 & 10 & $\begin{array}{l}20 \text { s on } \\
10 \text { points }\end{array}$ & Crushing of the right sciatic nerve. & On the sciatic nerve. & $\begin{array}{l}\text { 1st Application } 24 \mathrm{~h} \\
\text { after surgery; } 7,14 \text { and } \\
21 \text { successive days. }\end{array}$ & $\begin{array}{l}\text { LLLT increased the expression of } \\
\text { mRNA and the factors BDNF } \\
\text { and NGF after } 14 \text { days and } \\
\text { maximum expression was } \\
\text { observed on the 21st day. }\end{array}$ \\
\hline $\begin{array}{l}\text { Hsieh et al. } \\
{[47]}\end{array}$ & $\begin{array}{c}\text { GaAlAs } \\
\text { (Aculas-Am series, } \\
\text { Multi-channel laser } \\
\text { system; Konftec, } \\
\text { Taipei, Taiwan) }\end{array}$ & $660 / \approx 0.2$ & 30 & 9 & $\begin{array}{l}60 \text { s per point; } \\
4 \text { points }\end{array}$ & $\begin{array}{l}\text { Lesion on the sciatic nerve with } 4 \\
\text { ligatures, using chromic suture } 4-0 .\end{array}$ & On the surgery site. & $\begin{array}{l}\text { Application 7th } \\
\text { post-operatory, during } \\
7 \text { successive days. }\end{array}$ & $\begin{array}{l}\text { LLLT improved functional index, } \\
\text { decreased HIF-1a, TNF-a, and } \\
\text { IL-1b, increased VEGF, NGF, and } \\
\text { S100, reduced tissue ischemia } \\
\text { and inflammation, helped the } \\
\text { nerve recovery. }\end{array}$ \\
\hline Sene et al. [48] & $\begin{array}{l}\text { GaAsAl (Physiolux } \\
\text { Dual, BIOSET, Rio } \\
\text { Claro, Brazil) }\end{array}$ & $830 / 0.02$ & 30 & $\begin{array}{c}5 \\
10 \\
20\end{array}$ & $\begin{array}{l}\text { Maximum } \\
\text { time of } \\
\text { application } \\
\text { was } 40 \mathrm{~s}\end{array}$ & Crushing of the right fibular nerve. & $\begin{array}{l}\text { Application fibular } \\
\text { nerve region. }\end{array}$ & $\begin{array}{l}\text { Application } \\
\text { immediately after the } \\
\text { lesion, during } 21 \\
\text { successive days. }\end{array}$ & $\begin{array}{l}\text { LLLT simulation group obtained } \\
\text { a larger nerve transverse area; } \\
\text { group } 10 \mathrm{~J} / \mathrm{cm}^{2} \text { obtained higher } \\
\text { density of the fiber. LLLT did not } \\
\text { speed up nerve recovery. }\end{array}$ \\
\hline Dias et al. [49] & $\begin{array}{l}\text { GaAlAs (Mm Twin } \\
\text { Laser Optics, São } \\
\text { Carlos, Brazil) }\end{array}$ & $780 / 0.4$ & 30 & 15 & $\begin{array}{l}20 \text { s per point; } \\
3 \text { points }\end{array}$ & $\begin{array}{l}\text { Latex protein (F1) on lesion per } \\
\text { crushing of sciatic nerve. }\end{array}$ & $\begin{array}{l}\text { On the surgery site, sciatic } \\
\text { nerve. }\end{array}$ & $\begin{array}{l}\text { Application per } 6 \\
\text { sessions on } \\
\text { alternate days. }\end{array}$ & $\begin{array}{l}\text { LLLT associated to the F1 protein } \\
\text { did not present positive results } \\
\text { and did not potentialize the } \\
\text { effects of this protein. }\end{array}$ \\
\hline
\end{tabular}




\section{Discussion}

With the evolution of the technology in the health field and the evolution of the adjunct methods for rehabilitation and functional restoration of injured nerves [3,6-9], the PBMT has shown a wide range of benefits with clinical relevance. Thus, the aim of this research was to carry out a review of the scientific papers published in the last 10 years in order to verify the relation of PBMT in the regeneration of injured peripheral nerves. Regarding the varied benefits of PBMT, the highlight is the reduction of regeneration time and the aid in nerve function.

Among the effects of PBMT on nerve injury, it was verified that the laser minimized the side effects of bupivacaine on the nerve and on the muscle [29], potentiated the process of nerve regeneration observed by morpho-quantitative analysis of the axons and of the nerve fibers $[2,3,19,30-35]$, in addition to assisting muscular reinnervation [36].

Photobiomodulation in the nerve injury was also related to a decrease in inflammatory cytokine levels, in pain, and to the facilitation of neural regeneration, demonstrated by the levels of TNF-a, IL-1b, and GAP-43 [32,37].

The functional analysis evidenced the evolution of functional recovery associated with PBMT $[6,7,34,38,39]$. Marcolino et al. [40] found a functional recovery with both $40 \mathrm{~J} / \mathrm{cm}^{2}$ and $80 \mathrm{~J} / \mathrm{cm}^{2}$ $(830 \mathrm{~nm})$, Akgul; Gulsoy; Gulcur [41] also scored improvement in functionality with late application PBMT (650 nm) (7 days after injury), as well as Medalha et al. [35] at $660 \mathrm{~nm}$ at $50 \mathrm{~J} / \mathrm{cm}^{2}$. PBMT 660 $\mathrm{nm}, 10 \mathrm{~J} / \mathrm{cm}^{2}$, or $60 \mathrm{~J} / \mathrm{cm}^{2}$ accelerated neuromuscular recovery when compared to $780 \mathrm{~nm}$ and 830 nm PBMT [42]. Differently, dos Reis et al. [43] observed that PBMT significantly altered morphometry (myelin sheath thickness values) but did not interfere with the functionality.

Yang et al. [44], when associating PBMT with MSC, demonstrated a better electrophysiological function, immunoreactivity of S100, and fewer inflammatory cells. de Oliveira Martins et al. [45] demonstrated that PBMT $(904 \mathrm{~nm})$ had better nociception, greater expression of neural growth factor (NGF) 53\% and neurotrophic factor expression (BDNF) 40\%. As seen, Gomes; Dalmarco; André [46] evidenced that PBMT $(632.8 \mathrm{~nm})$ increased mRNA expression, BDNF and NGF factors after 14 days and maximum expression was observed on day 21. PBMT $(660 \mathrm{~nm})$ improved functional index, reduced HIF-1a, TNF-a, and IL-1b, elevated VEGF, NGF, and S100, and decreased tissue ischemia and inflammation [47]. Sene et al. [48] $(830 \mathrm{~nm})$ observed that PBMT did not accelerate nerve recovery and the study by Dias et al. [49] when associating PBMT $(780 \mathrm{~nm})$ with latex protein also did not find positive results.

The effects of PBMT on nerve damage were verified in the sciatic nerve in 17 articles [19,30-36,38,39,41-44,46,47,49], facial nerve in $3[3,6,7]$, fibular nerve in 2 articles [40,48], and vagus nerve [2], accessory nerve [29], alveolar nerve [45], and dorsal root [37] in one article each. Of the 26 articles inserted in this review, it was observed that 14 [19,31,32,37-42,44-46,48,49] presented compression as nerve damage (crushing) and $11[2,3,6,7,30,33-36,43,47]$ articles evaluated the effects of PBMT on neurotmeses, which is the worst type of nerve injury.

It has been observed that the diversity of PBMT application protocols in nerve lesions is large, with the wavelength varying from 632.8 to $904 \mathrm{~nm}$, a varied range of energy and energy density, in addition to the time of application, despite the similarity in the type of lesion targeted in each experiment. As shown, the infrared spectrum has good experimental results. The red spectrum (600 to $700 \mathrm{~nm}$ ) [50] was seen in 15 studies with satisfactory morphological and electrophysiological results, immunological factors, and tissue markers [2,30,31,33-36,38,39,41-44,46,47]. It was also possible to verify the lack of standardization in relation to the application protocols, noting that 6 studies were discarded due to lack of data information regarding energy density and time of application of PBMT.

In a general critical analysis of the articles for the detailed study, a consensus was observed on the effectiveness of PBMT, with the use of low-level laser therapy on the improvement of the morphological and morphometric aspects of the regenerated peripheral nerve, as well as on the reduction of events inflammatory and painful sensitivity, providing faster and higher quality functional recovery [51,52]. 
In the perspective of new fronts of study, in the last decade, optogenetic and chemogenetic techniques have been used more frequently in the investigation of neuronal circuits, as well as in the study of non-neuronal cells in the brain and peripheral nerves. Optogenetics is effective in generating patterns that mimic neuron responses using a pulse generator that produces lights with different frequencies and pulse durations. Photostimulation can be performed in different subcellular regions, being useful for the study of neuronal circuits in the brain. Chemogenetics are less invasive in animal experiments and do not require the installation of a fiber optic cable into the brain or the connection of the cable to a light source, such as a laser or a light emitting diode (LED).

\section{Conclusions}

At the end of the present study, it can be seen that the data presented in the current articles helped us to understand the beneficial and helpful effects of photobiomodulation on regeneration and functionality after nerve injury. In spite of the great variety of parameters presented, great results were observed, mainly when related to the faster nervous regeneration process.

Conflicts of Interest: The authors declare that they have no conflicts of interest.

\section{References}

1. Angeletti, P.; Pereira, M.D.; Gomes, H.C.; Hino, C.T.; Ferreira, L.M. Effect of low-level laser therapy (GaAlAs) on bone regeneration in midpalatal anterior suture after surgically assisted rapid maxillary expansion. Oral Surg. Oral Med. Oral Pathol. Oral Radiol. Endod. 2010, 109, e38-e46. [CrossRef] [PubMed]

2. Buchaim, R.L.; Andreo, J.C.; Barraviera, B.; Ferreira Junior, R.S.; Buchaim, D.V.; Rosa Junior, G.M.; de Oliveira, A.L.; de Castro Rodrigues, A. Effect of low-level laser therapy (LLLT) on peripheral nerve regeneration using fibrin glue derived from snake venom. Injury 2015, 46, 655-660. [CrossRef] [PubMed]

3. Buchaim, D.V.; de Castro Rodrigues, A.; Buchaim, R.L.; Barraviera, B.; Junior, R.S.; Junior, G.M.; Bueno, C.R.; Roque, D.D.; Dias, D.V.; Dare, L.R.; et al. The new heterologous fibrina sealant in combination with low-level laser therapy (LLLT) in the repair of the buccal branch of the facial nerve. Lasers Med. Sci. 2016, 31, 965-972. [CrossRef] [PubMed]

4. De Oliveira Gonçalves, J.B.; Buchaim, D.V.; de Souza Bueno, C.R.; Pomini, K.T.; Barraviera, B.; Júnior, R.S.F.; Andreo, J.C.; de Castro Rodrigues, A.; Cestari, T.M.; Buchaim, R.L. Effects of low-level laser therapy on autogenous bone graft stabilized with a new heterologous fibrin sealant. J. Photochem. Photobiol. B 2016, 162, 663-668. [CrossRef] [PubMed]

5. De Vasconcellos, L.M.; Barbara, M.A.; Rovai, E.S.; de Oliveira França, M.; Ebrahim, Z.F.; de Vasconcellos, L.G.; Porto, C.D.; Cairo, C.A. Titanium scaffold osteogenesis in healthy and osteoporotic rats is improved by the use of low-level laser therapy (GaAlAs). Lasers Med. Sci. 2016, 31, 899-905. [CrossRef] [PubMed]

6. Buchaim, D.V.; Andreo, J.C.; Ferreira Junior, R.S.; Barraviera, B.; Rodrigues, A.C.; Macedo, M.C.; Rosa Junior, G.M.; Shinohara, A.L.; Santos German, I.J.; Pomini, K.T.; et al. Efficacy of Laser Photobiomodulation on Morphological and Functional Repair of the Facial Nerve. Photomed. Laser Surg. 2017, 35, 442-449. [CrossRef] [PubMed]

7. Rosso, M.P.O.; Rosa Júnior, G.M.; Buchaim, D.V.; German, I.J.S.; Pomini, K.T.; de Souza, R.G.; Pereira, M.; Favaretto Júnior, I.A.; Bueno, C.R.S.; Gonçalves, J.B.O.; et al. Stimulation of morphofunctional repair of the facial nerve with photobiomodulation, using the end-to-side technique or a new heterologous fibrin sealant. J. Photochem. Photobiol. B 2017, 175, 20-28. [CrossRef] [PubMed]

8. Sulewski, J.G. Historical survey of laser dentistry. Dent. Clin. N. Am. 2000, 44, 717-752. [PubMed]

9. Ginani, F.; Soares, D.M.; Barreto, M.P.; Barboza, C.A. Effect of low-level laser therapy on mesenchymal stem cell proliferation: A systematic review. Lasers Med. Sci. 2015, 30, 2189-2194. [CrossRef] [PubMed]

10. Morries, L.D.; Cassano, P.; Henderson, T.A. Treatments for traumatic brain injury with emphasis on transcranial near-infrared laser phototherapy. Neuropsychiatr. Dis. Treat. 2015, 20, 2159-2175. [CrossRef]

11. Chang, W.D.; Wu, J.H.; Wang, H.J.; Jiang, J.A. Therapeutic outcomes of low-level laser therapy for closed bone fracture in the human wrist and hand. Photomed. Laser Surg. 2014, 32, 212-218. [CrossRef] [PubMed]

12. Gladsjo, J.A.; Jiang, S.I. Treatment of surgical scars using a 595-nm pulsed dye laser using purpuric and nonpurpuric parameters: A comparative study. Dermatol. Surg. 2014, 40, 118-126. [CrossRef] [PubMed] 
13. Keaney, T.C.; Tanzi, E.; Alster, T. Comparison of $532 \mathrm{~nm}$ potassium titanyl phosphate laser and $595 \mathrm{~nm}$ pulsed dye laser in the treatment of erythematous surgical scars: A randomized, controlled, Open-label study. Dermatol. Surg. 2016, 42, 70-76. [CrossRef] [PubMed]

14. Li, W.T.; Chen, H.L.; Wang, C.T. Effect of light emitting diode irradiation on proliferation of human bone marrow mesenchymal stem cells. J. Med. Biol. Eng. 2006, 26, 35-42.

15. Wu, Y.H.; Wang, J.; Gong, D.X.; Gu, H.Y.; Hu, S.S.; Zhang, H. Effects of low-level laser irradiation on mesenchymal stem cell proliferation: A microarray analysis. Lasers Med. Sci. 2012, 27, 509-519. [CrossRef] [PubMed]

16. Hashmi, J.T.; Huang, Y.Y.; Osmani, B.Z.; Sharma, S.K.; Naeser, M.A.; Hamblin, M.R. Role of low-level laser therapy in neurorehabilitation. PMER 2010, 2, 292-305. [CrossRef]

17. Yazdani, S.O.; Golestaneh, A.F.; Shafiee, A.; Hafizi, M.; Omrani, H.A.; Soleimani, M. Effects of low level laser therapy on proliferation and neurotrophic factor gene expression of human schwann cells in vitro. J. Photochem. Photobiol. B 2012, 107, 9-13. [CrossRef] [PubMed]

18. Martínez de Albornoz, P.; Delgado, P.J.; Forriol, F.; Maffulli, N. Non-surgical therapies for peripheral nerve injury. Br. Med. Bull. 2011, 100, 73-100. [CrossRef] [PubMed]

19. Ziago, E.K.; Fazan, V.P.; Iyomasa, M.M.; Sousa, L.G.; Yamauchi, P.Y.; da Silva, E.A.; Borie, E.; Fuentes, R.; Dias, F.J. Analysis of the variation in low-level laser energy density on the crushed sciatic nerves of rats: A morphological, quantitative, and morphometric study. Lasers Med. Sci. 2017, 32, 369-378. [CrossRef] [PubMed]

20. Gigo-Benato, D.; Geuna, S.; Rochkind, S. Phototherapy for enhancing peripheral nerve repair: A review of the literature. Muscle Nerve 2005, 31, 694-701. [CrossRef] [PubMed]

21. Rosso, M.P.O.; Buchaim, D.V.; Rosa Junior, G.M.; Andreo, J.C.; Pomini, K.T.; Buchaim, R.L. Low-Level Laser Therapy (LLLT) Improves the Repair Process of Peripheral Nerve Injuries: A Mini Review. Int. J. Neurorehabilit. 2017, 4, 260. [CrossRef]

22. Gigo-Benato, D.; Geuna, S.; de Castro Rodrigues, A.; Tos, P.; Fornaro, M.; Boux, E.; Battiston, B.; Giacobini-Robecchi, M.G. Low-power laser biostimulation enhances nerve repair after end-to-side neurorrhaphy: A double-blind randomized study in the rat median nerve model. Lasers Med. Sci. 2004, 19, 57-65. [CrossRef] [PubMed]

23. Rochkind, S.; Drory, V.; Alon, M.; Nissan, M.; Ouaknine, G.E. Laser phototherapy (780 nm), a new modality in treatment of long-term incomplete peripheral nerve injury: A randomized double-blind placebo-controlled study. Photomed. Laser Surg. 2007, 25, 436-442. [CrossRef] [PubMed]

24. Khullar, S.M.; Brodin, P.; Messelt, E.B.; Haanaes, H.R. The effects of low level laser treatment on recovery of nerve conduction and motor function after compression injury in the rat sciatic nerve. Eur. J. Oral Sci. 1995, 103, 299-305. [CrossRef] [PubMed]

25. Stainki, D.R.; Raiser, A.G.; Graça, D.L.; Becker, C.; Fernandez, G.M.S. Gallium arsenide (GaAs) laser radiation in the radial nerve regeneration submitted secondary to surgical repair. Braz. J. Vet. Res. Anim. Sci. 1999, 35, 37-40.

26. De Medinaceli, L.; Freed, W.J.; Wyatt, R.J. An index of the functional condition of rat sciatic nerve based on measurements made from walking tracks. Exp. Neurol. 1982, 7, 634-643. [CrossRef]

27. Gasparini, A.L.P.; Barbieri, C.H.; Mazzer, N. Correlation between different methods of gait functional evaluation in rats with ischiatic nerve crushing injuries. Acta Ortop. Bras. 2007, 15, 285-289. [CrossRef]

28. Moher, D.; Liberati, A.; Tetzlaff, J.; Altman, D.G.; The PRISMA Group. referred Reporting Items for Systematic Reviews and Meta-Analyses: The PRISMA Statement. PLoS Med. 2009, 6, e1000097. [CrossRef] [PubMed]

29. Alessi Pissulin, C.N.; Henrique Fernandes, A.A.; Sanchez Orellana, A.M.; Rossi, E.; Silva, R.C.; Michelin Matheus, S.M. Low-level laser therapy (LLLT) accelerates the sternomastoid muscle regeneration process after myonecrosis due to bupivacaine. J. Photochem. Photobiol. B 2017, 168, 30-39. [CrossRef] [PubMed]

30. Takhtfooladi, M.A.; Sharifi, D. A comparative study of red and blue light-emitting diodes and low-level laser in regeneration of the transected sciatic nerve after an end to end neurorrhaphy in rabbits. Lasers Med. Sci. 2015, 30, 2319-2324. [CrossRef] [PubMed]

31. Takhtfooladi, M.A.; Jahanbakhsh, F.; Takhtfooladi, H.A.; Yousefi, K.; Allahverdi, A. Effect of low-level laser therapy $\left(685 \mathrm{~nm}, 3 \mathrm{~J} / \mathrm{cm}^{2}\right)$ on functional recovery of the sciatic nerve in rats following crushing lesion. Lasers Med. Sci. 2015, 30, 1047-1052. [CrossRef] [PubMed] 
32. Wang, C.Z.; Chen, Y.J.; Wang, Y.H.; Yeh, M.L.; Huang, M.H.; Ho, M.L.; Liang, J.I.; Chen, C.H. Low-level laser irradiation improves functional recovery and nerve regeneration in sciatic nerve crush rat injury model. PLoS ONE 2014, 13, e103348. [CrossRef] [PubMed]

33. Shen, C.C.; Yang, Y.C.; Liu, B.S. Large-area irradiated low-level laser effect in a biodegradable nerve guide conduit on neural regeneration of peripheral nerve injury in rats. Injury 2011, 42, 803-813. [CrossRef] [PubMed]

34. Shen, C.C.; Yang, Y.C.; Liu, B.S. Effects of large-area irradiated laser phototherapy on peripheral nerve regeneration across a large gap in a biomaterial conduit. J. Biomed. Mater. Res. 2013, 101, 239-252. [CrossRef] [PubMed]

35. Medalha, C.C.; Di Gangi, G.C.; Barbosa, C.B.; Fernandes, M.; Aguiar, O.; Faloppa, F.; Leite, V.M.; Renno, A.C. Low-level laser therapy improves repair following complete resection of the sciatic nerve in rats. Lasers Med. Sci. 2012, 27, 629-635. [CrossRef] [PubMed]

36. Shen, C.C.; Yang, Y.C.; Huang, T.B.; Chan, S.C.; Liu, B.S. Low-Level Laser-Accelerated Peripheral Nerve Regeneration within a Reinforced Nerve Conduit across a Large Gap of the Transected Sciatic Nerve in Rats. Evid. Based Complement. Altern. Med. 2013, 2013, 175629. [CrossRef] [PubMed]

37. Chen, Y.J.; Wang, Y.H.; Wang, C.Z.; Ho, M.L.; Kuo, P.L.; Huang, M.H.; Chen, C.H. Effect of low level laser therapy on chronic compression of the dorsal root ganglion. PLoS ONE 2014, 9, e89894. [CrossRef] [PubMed]

38. Belchior, A.C.; dos Reis, F.A.; Nicolau, R.A.; Silva, I.S.; Perreira, D.M.; de Carvalho, P.D.T.C. Influence of laser $(660 \mathrm{~nm})$ on functional recovery of the sciatic nerve in rats following crushing lesion. Lasers Med. Sci. 2009, 24, 893-899. [CrossRef] [PubMed]

39. Barbosa, R.I.; Marcolino, A.M.; de Jesus Guirro, R.R.; Mazzer, N.; Barbieri, C.H.; de Cássia Registro Fonseca, M. Comparative effects of wavelengths of low-power laser in regeneration of sciatic nerve in rats following crushing lesion. Lasers Med. Sci. 2010, 25, 423-430. [CrossRef] [PubMed]

40. Marcolino, A.M.; Barbosa, R.I.; das Neves, L.M.; Mazzer, N.; de Jesus Guirro, R.R.; de Cássia Registro Fonseca, M. Assessment of functional recovery of sciatic nerve in rats submitted to low-level laser therapy with different fluences. An experimental study: Laser in functional recovery in rats. J. Hand Microsurg. 2013, 5, 49-53. [CrossRef] [PubMed]

41. Akgul, T.; Gulsoy, M.; Gulcur, H.O. Effects of early and delayed laser application on nerve regeneration. Lasers Med. Sci. 2014, 29, 351-357. [CrossRef] [PubMed]

42. Gigo-Benato, D.; Russo, T.L.; Tanaka, E.H.; Assis, L.; Salvini, T.F.; Parizotto, N.A. Effects of 660 and 780 nm low-level laser therapy on neuromuscular recovery after crush injury in rat sciatic nerve. Lasers Surg. Med. 2010, 42, 673-682. [CrossRef] [PubMed]

43. Dos Reis, F.A.; Belchior, A.C.G.; de Carvalho, P.T.C.; da Silva, B.A.; Pereira, D.M.; Silva, I.S.; Nicolau, R.A. Effect of laser therapy $(660 \mathrm{~nm})$ on recovery of the sciatic nerve in rats after injury through neurotmesis followed by epineural anastomosis. Lasers Med. Sci. 2009, 24, 741-747. [CrossRef] [PubMed]

44. Yang, C.C.; Wang, J.; Chen, S.C.; Hsieh, Y.L. Synergistic effects of low-level laser and mesenchymal stem cells on functional recovery in rats with crushed sciatic nerves. J. Tissue Eng. Regener. Med. 2016, 10, 120-131. [CrossRef] [PubMed]

45. De Oliveira Martins, D.; Martinez dos Santos, F.; Evany de Oliveira, M.; de Britto, L.R.; Benedito Dias Lemos, J.; Chacur, M. Laser therapy and pain-related behavior after injury of the inferior alveolar nerve: Possible involvement of neurotrophins. J. Neurotrauma 2013, 30, 480-486. [CrossRef] [PubMed]

46. Gomes, L.E.; Dalmarco, E.M.; André, E.S. The brain-derived neurotrophic factor, nerve growth factor, neurotrophin-3, and induced nitric oxide synthase expressions after low-level laser therapy in an axonotmesis experimental model. Photomed. Laser Surg. 2012, 30, 642-647. [CrossRef] [PubMed]

47. Hsieh, Y.L.; Chou, L.W.; Chang, P.L.; Yang, C.C.; Kao, M.J.; Hong, C.Z. Low-level laser therapy alleviates neuropathic pain and promotes function recovery in rats with chronic constriction injury: Possible involvements in hypoxia-inducible factor $1 \alpha$ (HIF-1 $\alpha)$. J. Comp. Neurol. 2012, 520, 2903-2916. [CrossRef] [PubMed]

48. Sene, G.A.; Sousa, F.F.; Fazan, V.S.; Barbieri, C.H. Effects of laser therapy in peripheral nerve regeneration. Acta Ortop. Bras. 2013, 21, 266-270. [CrossRef] [PubMed] 
49. Dias, F.J.; Issa, J.P.; Coutinho-Netto, J.; Fazan, V.P.; Sousa, L.G.; Iyomasa, M.M.; Papa, P.C.; Watanabe, I.S. Morphometric and high-resolution scanning electron microscopy analysis of low-level laser therapy and latex protein (Hevea brasiliensis) administration following a crush injury of the sciatic nerve in rats. J. Neurol. Sci. 2015, 349, 129-137. [CrossRef] [PubMed]

50. Moore, P.; Ridgway, T.D.; Higbee, R.G.; Howard, E.W.; Lucroy, M.D. Effect of wavelength on low-intensity laser irradiation-stimulated cell proliferation in vitro. Lasers Surg. Med. 2005, 36, 8-12. [CrossRef] [PubMed]

51. Bang, J.; Kim, H.Y.; Lee, H. Optogenetic and Chemogenetic Approaches for Studying Astrocytes and Gliotransmitters. Exp. Neurobiol. 2016, 25, 205-221. [CrossRef] [PubMed]

52. Iyer, S.M.; Vesuna, S.; Ramakrishnan, C.; Huynh, K.; Young, S.; Berndt, A.; Lee, S.Y.; Gorini, C.J.; Deisseroth, K.; Delp, S.L. Optogenetic and chemogenetic strategies for sustained inhibition of pain. Sci. Rep. 2016, 6, 30570. [CrossRef] [PubMed]

(C) 2018 by the authors. Licensee MDPI, Basel, Switzerland. This article is an open access article distributed under the terms and conditions of the Creative Commons Attribution (CC BY) license (http://creativecommons.org/licenses/by/4.0/). 